Check for updates

Cite this: Phys. Chem. Chem. Phys., 2018, 20, 19486

Received 11th June 2018 Accepted 3rd July 2018

DOI: $10.1039 / \mathrm{c} 8 \mathrm{cp} 03684 \mathrm{e}$

rsc.li/pccp

\title{
Bridge-bonded methylthiolate on Au(111) observed with the scanning tunneling microscope
}

\author{
Scott Holmes, ${ }^{a}$ Jianzhi Gao, ${ }^{b}$ Lin Tang, ${ }^{c}$ Fangsen Li, ${ }^{d}$ Richard E. Palmer ${ }^{\mathbb{D}}{ }^{\mathrm{e}}$ and \\ Quanmin Guo (D)*a
}

\begin{abstract}
We report the discovery of bridge-bonded methylthiolate, $\mathrm{SCH}_{3}$, along the step edges of the $\mathrm{Au}(111)$ surface. Real-space imaging with a scanning tunnelling microscope reveals the presence of bridgebonded $\mathrm{SCH}_{3}$ along both the [11̄0] and the [112] oriented step edges. The nearest neighbour distances of $\mathrm{SCH}_{3}$ along these steps are $2 a$ and $\sqrt{3} a$, respectively. The Au(111) terrace is covered with the usual $\mathrm{CH}_{3} \mathrm{SAuSCH}_{3}$ staples. The bridge-bonded alkanethiolate is expected to play a rather significant role in the formation of thiol-passivated Au nanoclusters because of the high fraction of atoms in similar low-coordination sites.
\end{abstract}

\section{Introduction}

Alkanethiol passivated gold surfaces and nanoclusters lead to a rich variety of surface reconstructions and, in the latter case, size-dependent electronic and optical properties. ${ }^{1-4}$ Due to their very small sizes, the determination of the structure of these nanoclusters has been a great challenge. ${ }^{5-12}$ Recent studies demonstrate that even for the magic number $\mathrm{Au}_{n}(\mathrm{SR})_{m}$ clusters, there is structural polymorphism. ${ }^{7,12}$ In contrast to naked $\mathrm{Au}$ nanoclusters, thiolate passivation does not just protect the clusters from interacting with other clusters or the surroundings, but also directly influences the structure of the clusters via the $\mathrm{S}-\mathrm{Au}$ bonding at the interface. ${ }^{13}$ The current view on the S-Au bonding at the thiolate-Au interface is based on the RS-Au-SR staple motif which has been found on both the surface of $\mathrm{Au}$ nanoclusters ${ }^{6}$ using X-ray diffraction and extended $\mathrm{Au}(111)$ surfaces by direct imaging with a scanning tunneling microscope (STM).${ }^{14-18}$ Variations of the RS-Au-SR staple motif, such as the dimeric ${ }^{13}$ and trimeric ${ }^{19}$ staple motifs, have also been introduced to explain the structure of passivated $\mathrm{Au}$ nanoclusters. An interesting, but less conventional, structural motif containing SR bridge-bonded to surface Au atoms was reported recently. ${ }^{11}$ This bridge-bonded SR, if existing on the surface of $\mathrm{Au}$ nanoclusters, is expected to have a great impact on our

\footnotetext{
${ }^{a}$ School of Physics and Astronomy, University of Birmingham, Edgbaston, Birmingham, B15 2TT, UK. E-mail: Q.Guo@bham.ac.uk; Tel: +44 1214144657

${ }^{b}$ School of Physics and Information Technology, Shaanxi Normal University, Xi'an 710119, China

${ }^{c}$ Department of Physics, Tsinghua University, Beijing, 100084, China

${ }^{d}$ Suzhou Institute of Nano-Tech and Nano-Bionics, SEID, Suzhou Industrial Park, Suzhou, Jiangsu Province, 215123, China

${ }^{e}$ College of Engineering, Swansea University, Bay Campus, Fabian Way, Swansea, SA1 $8 E N, U K$
}

understanding of the thiolate-Au interface. Herein we report the discovery of bridge-bonded SR on the extended $\mathrm{Au}(111)$ surface by imaging with the STM. The capability of STM to perform real space imaging with atomic resolution makes it a useful complementary technique to diffraction analysis, providing important local structural information at the atomic level.

\section{Methods}

A (111)-oriented Au single crystal is used for the experiment. The sample is cleaned by standard ion sputtering and thermal annealing cycles. The clean $\mathrm{Au}(111)$ is then exposed to dimethyldisulfide (DMDS) at room temperature to reach full surface coverage. This is followed by thermal annealing at $350 \mathrm{~K}$ to desorb some molecules so that the remaining molecules form the striped phase. ${ }^{18}$ After cooling to $77 \mathrm{~K}$, the sample is imaged with an Omicron low temperature STM (LT-STM) with an electrochemically etched tungsten tip.

\section{Results and discussion}

Fig. 1 shows an STM image of the Au(111) sample with $\sim 0.2$ monolayers (ML) of methylthiolate. On the flat (111) terraces, we find the usual rows of $\mathrm{CH}_{3} \mathrm{~S}-\mathrm{Au}-\mathrm{SCH}_{3}$ staples aligned in one of the $\langle 112\rangle$ directions. ${ }^{18}$ For each $\mathrm{CH}_{3} \mathrm{~S}-\mathrm{Au}-\mathrm{SCH}_{3}$ staple, highresolution STM images show three protrusions: two from the $\mathrm{CH}_{3}$ groups and one from the $\mathrm{Au}$ adatom ${ }^{15,18}$ with $\mathrm{S}$ not visible under normal imaging conditions. The inset in Fig. 1, for example, shows a row of four $\mathrm{CH}_{3} \mathrm{~S}-\mathrm{Au}-\mathrm{SCH}_{3}$ staples. The $\mathrm{Au}$ adatom becomes less visible for thiols with longer alkane chains. ${ }^{19,20}$ The distance between the two staples along the row in the [11ㄹ] direction is $\sqrt{3} a$. The distance between the two methyl groups 


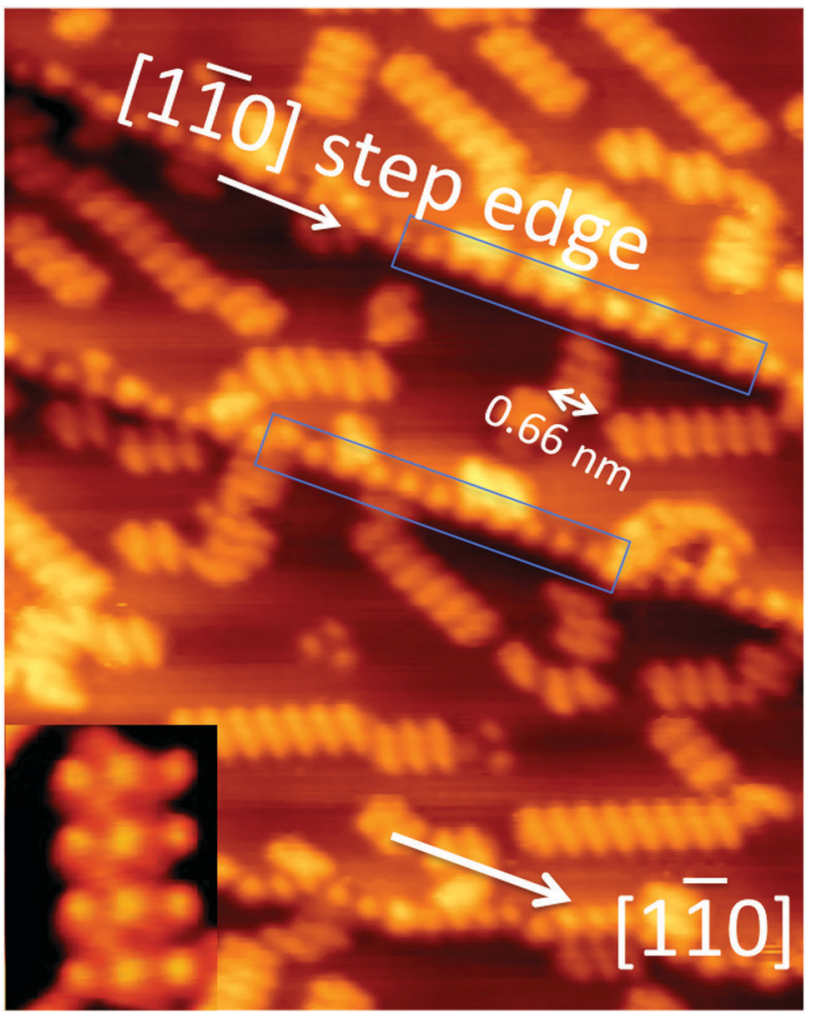

Fig. 1 STM image, $15 \mathrm{~nm} \times 20 \mathrm{~nm}$, acquired at $77 \mathrm{~K}$ from $\mathrm{Au}(111)$ with $0.2 \mathrm{ML}$ of methylthiolate. The two blue rectangular boxes highlight two segments of [11̄0] oriented step edges. The inset shows a high-resolution image where two $\mathrm{CH}_{3}$ groups and their associated Au adatoms in each staple are resolved.

within the same staple, measured to be $0.66 \mathrm{~nm}$, is a pure property of the staple, and has no specific relation to the lattice constant of gold. The occurrence of paired-spots, representing the two arms of RS-Au-SR, is a distinctive feature for the presence of RS-Au-SR staples on $\mathrm{Au}(111)$. In addition to the standard $\mathrm{CH}_{3} \mathrm{~S}-\mathrm{Au}-\mathrm{SCH}_{3}$ staples found on the terraces, we observe regularlyspaced spots along the step edges, organized in a different manner from those associated with the staples. In Fig. 1, two sections of the $[1 \overline{1} 0]$ oriented step edges are highlighted with blue rectangular boxes. These steps consist of close-packed Au atoms. Fig. 2a shows a faceted step edge consisting of segments of [110] steps. Fig. $2 \mathrm{~b}$ presents a height profile taken along the double-headed arrow, A-B. This allows an accurate measurement of the distance between the nearest neighboring spots along the step. The measured distance is $0.58 \pm 0.01 \mathrm{~nm}$ which is significantly smaller than the distance $(0.66 \mathrm{~nm})$ between the two methyl groups within one staple. Therefore, the spots along the [110] step are not paired. They are individual spots equally spaced along the step. $0.58 \pm 0.01 \mathrm{~nm}$ is consistent with $2 a$ where $a$ is the distance between the nearest neighboring Au atoms. Thus, we conclude that along the [11̄0] step there is one spot for every two $\mathrm{Au}$ atoms.

There are not many detailed studies on the organization of alkanethiolate along atomic steps on $\mathrm{Au}(111)$. Step decoration by methylthiolate on $\mathrm{Au}(111)$ has been reported on two previous

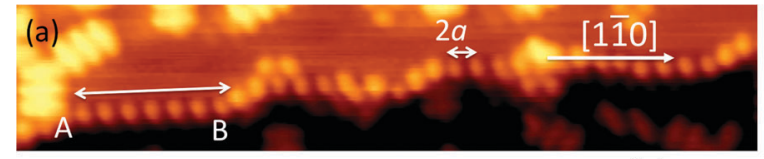

(b)
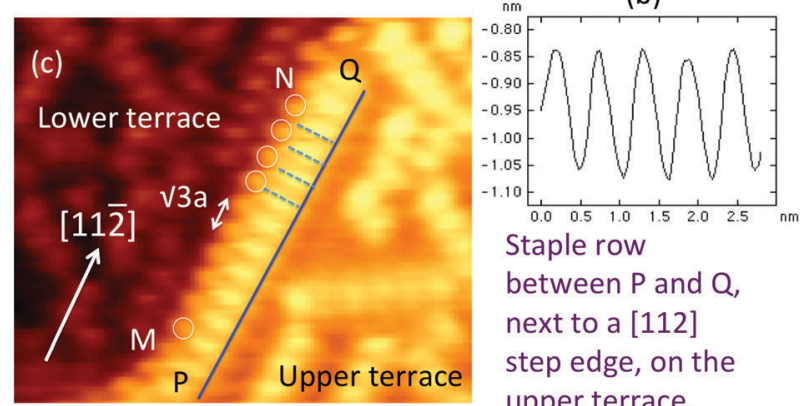

Staple row

between $\mathrm{P}$ and $\mathrm{Q}$, next to a [112] step edge, on the upper terrace.

Fig. 2 (a) Single row of spots observed along the [110] step edge. Spots are equally spaced at a distance of $2 a$. (b) Height profile taken along the line A-B. (c) Image of thiolate along a [112] step, $\mathrm{M}-\mathrm{N}$. There is a staple row along $P-Q$. Four dashed lines highlight four staples. Circles are used to show the locations of the non-pairing spots.

occasions ${ }^{17,21}$ with no structural model proposed. In an STM study of ethylthiolate on $\mathrm{Au}(111)$, Clair et al. reported the decoration of the $[1 \overline{1} 0]$ step edges by single thiolate rows. ${ }^{22}$ The single row of ethylthiolate reported by Clair $e t$ al. was assumed to have a similar bonding configuration to that occurring in a staple. However, the remarkable similarity between the ethylthiolate rows observed by Clair et al. and the methylthiolate rows shown in Fig. 1 and 2 suggests that they are the same type of species. The nearest neighbor distance between the ethylthiolates along the [11̄0] step is $0.55 \mathrm{~nm}^{22}$ which is also very close to the distance $(0.58 \mathrm{~nm})$ measured in this study for the methylthiolates allowing for measurement uncertainties. The distance measured in our study is calibrated against the distance $(0.5 \mathrm{~nm})$ between staples along a staple row and it has an uncertainty of $\pm 0.01 \mathrm{~nm}$.

In Fig. 2c, we show a $[11 \overline{2}]$ oriented step. There is a $\mathrm{CH}_{3} \mathrm{~S}-$ $\mathrm{Au}-\mathrm{SCH}_{3}$ staple row sitting on the upper terrace at this step edge, along the direction $\mathrm{P}-\mathrm{Q}$. This staple row has the same appearance as all other staple rows on the terrace. Interestingly, one can see a single row of spots, $\mathrm{M}-\mathrm{N}$, right next to the staple row, $\mathrm{P}-\mathrm{Q}$. A few circles are drawn onto the image to assist the eye to locate this single row of spots. Again, here we have a row of spots showing no signs of pairing. These spots along $\mathrm{M}-\mathrm{N}$ are regularly spaced at a distance of $0.50 \pm 0.01 \mathrm{~nm}$, which corresponds to $\sqrt{3} a$. The spots along M-N appear $0.07 \pm 0.01 \mathrm{~nm}$ lower than the methyl groups along the $\mathrm{P}-\mathrm{Q}$ staple row, and are $0.16 \pm 0.01 \mathrm{~nm}$ higher than the methyl groups on the lower terrace. This gives further evidence that the spots along the step edges are different from those found either on the upper or the lower terraces. For the spots along both the $[1 \overline{1} 0]$ and $[11 \overline{2}]$ step edges, the distance between the neighboring spots is determined by the geometry of the gold substrate: $2 a$ along the [11 10$]$ direction and $\sqrt{3} a$ along the $[1 \overline{1} 0]$ direction. Based on these observations and the above discussion, we come to the conclusion that each spot along the step edge corresponds to a single $\mathrm{SCH}_{3}$ species bonded to Au atoms along the step, and there is 


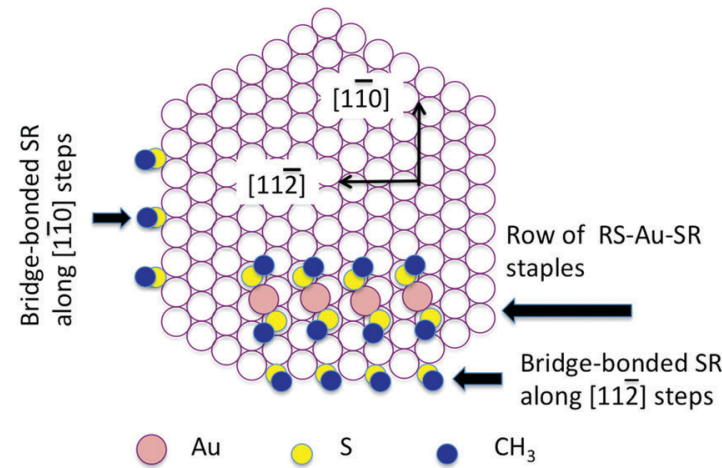

Fig. 3 Structural model illustrating how $\mathrm{SCH}_{3}$ is bonded to $\mathrm{Au}$ atoms along step edges. Along the [112]] step, there are two bridging sites available for each $\mathrm{SCH}_{3}$. Binding of $\mathrm{SCH}_{3}$ at one of the bridging sites may be more favorable than the other. In the figure, $\mathrm{SCH}_{3}$ is placed arbitrarily at one of the bridging sites.

no association of two adjacent $\mathrm{SCH}_{3}$ groups such as those found within a staple.

In Fig. 3, we present a structural model showing the proposed bonding of the $\mathrm{SCH}_{3}$ group to $\mathrm{Au}$ atoms at steps. Considering the nearest neighbor distances and the likelihood that the $\mathrm{SCH}_{3}$ group would occupy a high symmetry site, there are only two possible bonding sites for $\mathrm{SCH}_{3}$ along the [110] step: monodentate bonding with $\mathrm{S}$ bound to a single $\mathrm{Au}$ atom or bidentate bonding with $\mathrm{S}$ bridging between two $\mathrm{Au}$ atoms. Adsorption energetics suggests that the bidentate form is much more favored. ${ }^{23}$ Along the [1ㅣㅣ step, the shortest distance possible between two bridge-bonded $\mathrm{SCH}_{3}$ groups is thus $2 a$, as $1 a$ is too short a distance to accommodate two neighboring methyl groups. This bonding scheme is similar to the bonding of $\mathrm{SCH}_{3}$ within the staple, where $\mathrm{S}$ interacts directly with two gold atoms: one is the Au adatom in the staple and the other the $\mathrm{Au}$ atom within the top layer of $\mathrm{Au}(111)$. Along the [110] step, the $\mathrm{S}$ atom is bonded to two $\mathrm{Au}$ atoms in identical positions and the $\mathrm{S}-\mathrm{C}$ bond is expected to be perpendicular to the step edge. This makes the $\mathrm{CH}_{3}$ group along the step appear at a height between the methyl groups on the upper terrace and those on the lower terrace.

On the (111) plane of fcc metals, there are two types of [110] oriented steps: (i) a step presenting a $\{111\}$ microfacet and (ii) a step presenting a $\{100\}$ microfacet. ${ }^{24}$ The two types of steps are energetically different from the $\{111\}$-faceted step having lower energy. This energy difference is expected to influence molecular adsorption. We do not know which type of step is the one observed in Fig. 1, although the two [110] steps in the figure belong to the same type. It would be of interest to find out if thiolate interacts differently with the two types of steps. In a previous study using a zero-gradient stepped surface, we have shown that the adsorption of $\mathrm{C}_{60}$ molecules on $\mathrm{Au}(111)$ exhibits clear step type dependence. $^{25}$

The $[11 \overline{2}]$ step has a zig-zag structure exposing micro-faceted [110] steps. For the reason as discussed above, we have alternating $\{111\}$ - and $\{100\}$-faceted steps along the [112] direction. Each $\{111\}$ - or $\{100\}$-faceted step is only two-atoms long.
The $\mathrm{SCH}_{3}$ group hence has a choice between the two types of steps. In Fig. 3, we have arbitrarily put $\mathrm{SCH}_{3}$ onto one of the bridging sites. As can be seen in Fig. 3, the horizontal step at the bottom of the diagram is a [112] $]$ oriented step. Along this step, $\mathrm{SCH}_{3}$ can find identical bridge-bonding sites to those presented by a [110] oriented step. Applying the same bridgebonding scheme for the [110] step, we can generate a row of $\mathrm{SCH}_{3}$ parallel to the $[11 \overline{2}]$ direction with the nearest neighboring $\mathrm{SCH}_{3}$ groups separated by $\sqrt{3} a$. The model in Fig. 3 is in good agreement with what is observed in STM images. For example, the position of $\mathrm{SCH}_{3}$ attached to the [112] step relative to the position of the first staple row on the upper terrace is correctly given by the model.

From the STM images, we find that the step edges are almost fully covered with thiolate while the coverage on the terrace is lower. This suggests preferential binding of thiolate at step edges in competition with the formation of the RS-Au-SR staple on terraces. Indeed, the bridge-bonded species have a higher thermal stability than the staples. We find that at $210 \mathrm{~K}$ when the staple rows on the terraces are fully disintegrated to form a liquid-like layer, the bridge-bonded species along the steps remain static. This finding is important when one attempts to explain the formation of staples that require the supply of Au adatoms. Since adatoms on surfaces usually come from steps, the passivation of steps by bridge-bonded thiolate may play a key role in the formation of SAMs on $\mathrm{Au}(111)$. At RT, no ordered $\mathrm{CH}_{3} \mathrm{SAuSCH}_{3}$ staple rows are observed on $\mathrm{Au}(111)$. Instead, the STM image shows a disordered layer indicating rapid movement of adsorbed species. $\mathrm{CH}_{3} \mathrm{SAuSCH}_{3}$ can break apart into $\mathrm{CH}_{3} \mathrm{SAu}$ and $\mathrm{SCH}_{3}$ or $2 \mathrm{SCH}_{3}$ and $\mathrm{Au}$. There is a possibility that when $\mathrm{SCH}_{3}$ moves close to a step already occupied by bridge-bonded thiolate, it may combine with a thiolate at the step to form $\mathrm{CH}_{3} \mathrm{SAuSCH}_{3}$ which moves away from the step into the terrace. If the temperature is not sufficiently high, the above proposed reaction step does not occur and the step is protected from being etched. For alkanethiol molecules with long alkane chains, the diffusion of the rather bulky molecules at RT is slow on $\mathrm{Au}(111)$. Thus, $\mathrm{Au}$ atoms might get extracted locally from the flat atomic terraces and hence etch pits are formed. For short chain alkanethiols deposited onto $\mathrm{Au}(111)$ using their vapors under vacuum, etch pits in general do not appear. However, etch pits can form if the $\mathrm{Au}(111)$ is dipped into a liquid solution of short chain thiols. This is because the extraction of $\mathrm{Au}$ atoms from steps is too slow in comparison to the uptake of the thiol molecules in such an environment.

Bridge-bonded thiolate is likely to be a more common species considering that there is now evidence for the presence of both methylthiolate and ethylthiolate on $\mathrm{Au}(111)$, as well as on the surface of gold nano-particles. ${ }^{11}$ In the following, we provide some additional information on the assembly of thiolate in the vicinity of steps on $\mathrm{Au}(111)$. Data presented below are from the $\mathrm{Au}(111)$ surface fully covered with a layer of mixed methylthiolate and propylthiolate. The sample was prepared by exposing a (111) oriented $\mathrm{Au}$ film to methyl-propyl-disulfide, $\mathrm{CH}_{3} \mathrm{~S}-\mathrm{S}\left(\mathrm{CH}_{2}\right)_{2} \mathrm{CH}_{3}$, at RT with STM imaging also conducted at RT. No thermal annealing was conducted between deposition 
and imaging. The initial motivation for studying the mixed molecular layer came from our earlier work with methyl- and ethyl-thiolate on $\mathrm{Au}(111),{ }^{17}$ where we demonstrated that both methyl- and ethyl-thiolate form a $3 \times 4$ phase at saturation coverage, instead of the well-documented $(\sqrt{ } 3 \times \sqrt{ } 3)-R 30^{\circ}$ phase. By depositing $\mathrm{CH}_{3} \mathrm{~S}-\mathrm{S}\left(\mathrm{CH}_{2}\right)_{2} \mathrm{CH}_{3}$ onto $\mathrm{Au}(111)$, we were hoping to observe domain-separated $3 \times 4$ methylthiolate and $(\sqrt{ } 3 \times \sqrt{ } 3)-R 30^{\circ}$ propylthiolate on the same sample. But it was only discovered later that propylthiolate also forms the $3 \times 4$ phase, albeit with a lower degree of crystallinity. ${ }^{20}$

Methylthiolate and propylthiolate tend to phase separate on $\mathrm{Au}(111)$, but the phase separation is not extensive and there are regions of the sample having a mixture of both. ${ }^{26}$ Whilst both ethyl- and propyl-thiolate monolayers are stable at RT, ${ }^{19,20}$ the mixed methyl- and propyl-thiolate layer is rather fluidic with constant molecular movement. This is mainly due to the contribution of the methylthiolate. Moving from methylthiolate to ethyl and propylthiolate, the increase of the alkane chain length offers some extra stability to the adsorbate due to the contribution of the van der Waals interaction. In addition to the van der Waals interaction, there is also a possibility that the methylthiolate staple is intrinsically less stable. The reason for nonobservance of a stable structure at RT for a full layer of methylthiolate on $\mathrm{Au}(111)$ is likely that the $\mathrm{CH}_{3} \mathrm{SAuSCH}_{3}$ staple breaks down to $\left(\mathrm{CH}_{3} \mathrm{~S}\right)^{-}$(a negative-ion-like species) and Au. We expect an abrupt change in the stability of the negative-ion-like species when the number of carbon in the thiolate increases from one to two or more. A relatively stable adsorbate structure is found along step edges as shown in Fig. 4 which is an STM image acquired at RT from an area enriched with propylthiolate. Due to the mixing of methylthiolate and propylthiolate on the surface, it is not easy to identify which feature is due to

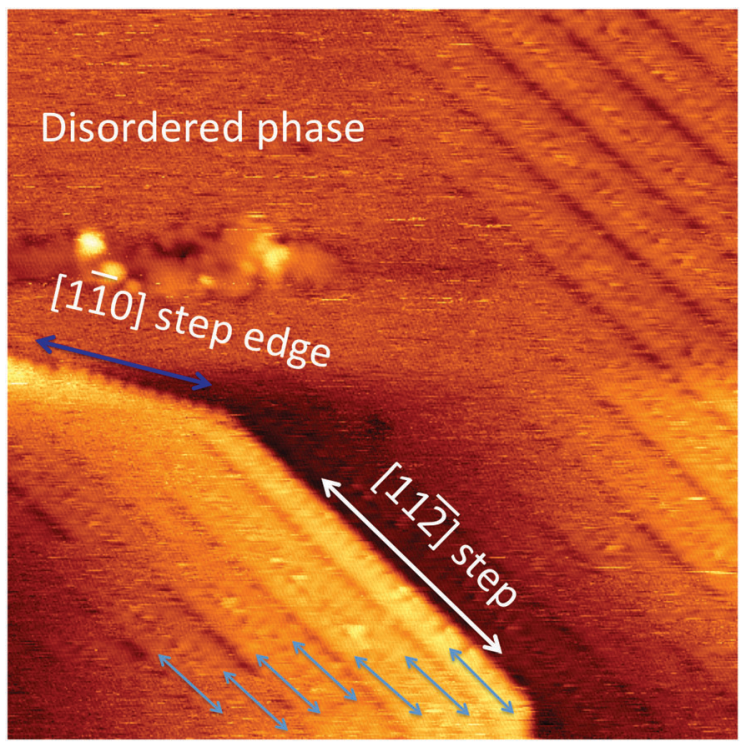

Fig. 4 STM image, $25 \mathrm{~nm} \times 25 \mathrm{~nm}$, showing thiolates along step edges. The image shows Au(111) covered with a layer of mixed methylthiolate and propylthiolate. The combined coverage is close to the saturation value. This image shows a propylthiolate-rich region. methylthiolate and which is due to propylthiolate. However, a methylthiolate-rich region is expected to be completely disordered at RT. Since relatively stable staple rows are observed in Fig. 4, we believe that this region consists of mainly propylthiolate. The image consists of an upper terrace and a lower terrace with steps in between. There is a segment of the [110] oriented step and a segment of the $[11 \overline{2}]$ oriented step. It can be seen that the [110] step is decorated by bridge-bonded thiolates with a regular spacing of $0.58 \mathrm{~nm}$. There is a staple row of RS-Au-SR along the [112]] step on the upper terrace. In this image, the resolution is not high enough to resolve the bridgebonded species along the [11/2] step. From our observation, the first row of staples on the upper terrace is formed at the step edge. On some narrow terraces, no ordered structure exists apart from one single row of staples pinned to the [112̄] step edge. More rows are formed subsequently during scanning and eventually a whole terrace can be covered with the staple rows. There is also a region of the disordered phase on the lower terrace. As the area is scanned continuously, the striped phase is observed to grow by consuming the disordered phase. Both the $[1 \overline{1} 0]$ and $[11 \overline{2}]$ oriented steps are stable. However, not all steps are stable. Fig. 5 shows an STM image covering a larger area than that of Fig. 4. In Fig. 5, we can see that near the upper right hand corner, there is an area with a high density of [11̄] oriented steps. All the [112]] oriented steps have a common feature that there is a staple row running along the step edge on the upper terrace. Areas marked by white rectangles consist of steps not along any major crystallographic directions. The hairy/spiky appearance of these steps indicates that there are atoms/molecules constantly entering as well as leaving these steps. The terraces next to these irregular and changing steps

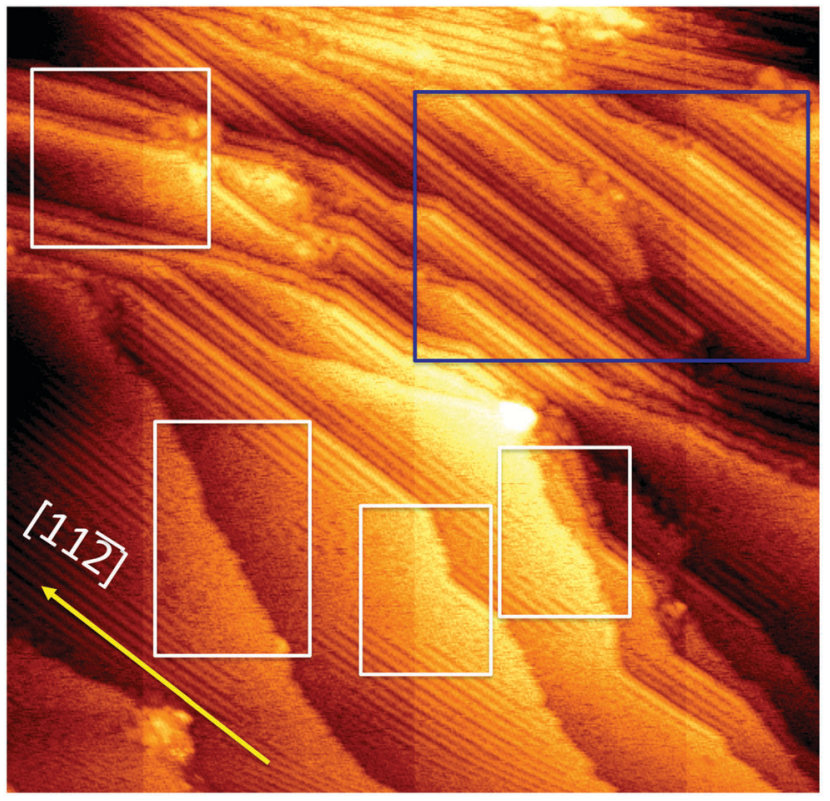

Fig. 5 STM image, $75 \mathrm{~nm} \times 75 \mathrm{~nm}$, showing regular staple rows in regions with parallel [11ㄹ] steps. Steps not along the major crystallographic directions appear to be less stable and thiolates near such irregular steps are in a disordered state. 
do not have an ordered structure. It is likely that these irregular steps are the providers of gold atoms for the formation of the staples. The $[11 \overline{2}]$ oriented steps are not the stable steps on the clean $\mathrm{Au}(111)$ surface. However, they seem to be abundant on the thiolate covered surface. These [112]] oriented steps are relatively long and very straight suggesting a significant stabilizing effect from the step-bound thiolates. From the structural model of Fig. 3, it can be seen that the [112] step is more open than the $[1 \overline{1} 0]$ step. Au atoms along the $[11 \overline{2}]$ step have a lower coordination number. This may enhance the bonding to the thiolates as a result of a higher charge density offered by the $[11 \overline{2}]$ step. It is also possible that many of the [112] oriented steps are transformed from irregular steps mediated by the diffusing SR.

Since the discovery of the RS-Au-SR staple motif on both the surfaces of Au nano-clusters and the $\mathrm{Au}(111)$ crystal surface, ${ }^{6,15}$ numerous studies have demonstrated that such a staple motif applies almost universally to all small Au clusters. However, recent work reports the finding of bridge-bonded thiolate on the (001) surface of a tetragonal Au nanocrystal. ${ }^{9}$ The direct observation of bridge-bonded thiolate along step edges on $\mathrm{Au}(111)$ is significant for the understanding of the structures of larger Au clusters with a fcc lattice. Such clusters have exposed (111) and (100) facets with edges similar to those found on planar $\mathrm{Au}(111)$ surfaces. Even for small clusters which are not fcc, there is still a possibility of forming bridge-bonded SR along the edges of the cluster surfaces.

It is interesting to speculate whether alkylthiolates with longer chains such as butylthiolate would form the same kind of bridge-bonded species along step edges on $\mathrm{Au}(111)$. It is known that the van der Waals interaction between the alkyl chains plays an important role in the self-assembly of akylthiolate monolayers on $\mathrm{Au}(111)$. Most strikingly, at maximum coverage, thiolates with three or fewer carbon atoms ${ }^{16,18-20}$ form the $3 \times 4$ phase, while thiolates with more than three carbon atoms in the chain take a different $2 \sqrt{3} a \times 3$-rect. structure. ${ }^{27,28}$ We expect that the formation of the bridge-bonded species along step edges may also show some chain length dependence.

\section{Conclusions}

To summarize, we have found clear evidence of bridge-bonded

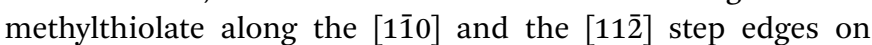
$\mathrm{Au}(111)$. There is also some indication of bridge-bonded propylthiolate from the experiment involving mixed methyl- and propylthiolate, but further confirmation of such species is needed in future studies. This bridge-bonded SR is expected to play an important role in the assembly of SAMs on both the planar (111) surface and on the surfaces of gold nano-particles. More work is needed in this area to search for bridge-bonded SR at saturation coverage and also for thiolates with longer chains. Because of the importance of surface steps in providing free-diffusing $\mathrm{Au}$ atoms, understanding the nature of step decoration by bridge-bonded thiolate would be an important achievement in terms of a better explanation of alkanethiol self-assembly.

\section{Conflicts of interest}

There are no conflicts to declare.

\section{Acknowledgements}

We thank the Engineering and Physical Sciences Research Council of the United Kingdom for financial support.

\section{References}

1 M. Zhu, C. M. Aikens, F. J. Hollander, G. C. Schatz and R. Jin, Correlating the crystal structure of a thiol-protected Au-25 cluster and optical properties, J. Am. Chem. Soc., 2008, 130, 5883.

2 H. C. Weissker, et al., Information on quantum states pervades the visible spectrum of the ubiquitous $\mathrm{Au}_{144} \mathrm{SR}_{60}$ gold nanocluster, Nat. Commun., 2014, 5, 3785.

3 R. Philip, P. Chantharasupawong, H. F. Qian, R. C. Jin and J. Thomas, Evolution of nonlinear optical properties: From gold atomic clusters to plasmonic nanocrystals, Nano Lett., 2012, 12, 4661.

4 Y. Negishi, et al., A critical size for emergence of nonbulk electronic and geometric structures in dodecanethiolateprotected Au clusters, J. Am. Chem. Soc., 2014, 137, 1206-1212.

5 A. Dass, S. Theivendran, P. R. Nimmala, C. Kumara, V. R. Jupally, A. Fortunelli, G. Barcaro, X. Zuo and B. C. Noll, Au1 ${ }_{33}$ (SPh-tBy) $5_{2}$ nanomolecules: X-ray crystallography, optical, electrochemical, and theoretical analysis, J. Am. Chem. Soc., 2015, 137, 4610.

6 P. D. Jadzinsky, G. Careo, C. J. Ackerson, D. A. Bushnell and R. D. Kornberg, Structure of a thiol monolayer-protected gold nanoparticle at 1.1 angstrom resolution, Science, 2007, 130, 3754 .

7 K. M. O. Jensen, P. Juhas, M. A. Tofanelli, C. L. Heinecke, G. Vauhgan, C. J. Ackerson and S. J. L. Billinge, Polymorphism in magic-sized $\mathrm{Au}_{144} \mathrm{SR}_{60}$ clusters, Nat. Commun., 2016, 7, 11859.

8 C. Zeng, Y. Chen, K. Iida, K. Nobusada, K. Kirschbaum, K. J. Lambright and R. Jin, Gold quantum boxes: on the periodicities and the quantum confinement in the $\mathrm{Au}_{28}$, $\mathrm{Au}_{36}, \mathrm{Au}_{44}$ and $\mathrm{Au}_{52}$ magic series, J. Am. Chem. Soc., 2016, 138, 3950.

9 C. Zeng, C. Liu, Y. Chen, N. Rosi and R. Jin, Atomic structure of self-assembled monolayer of thiolate on a tetragonal $\mathrm{Au}_{92}$ nanocrystal, J. Am. Chem. Soc., 2016, 138, 8710.

10 H. W. Dong, L. W. Liao and Z. K. Wu, Two-way transformation between fcc- and non-fcc-structured gold nanoclusters, J. Phys. Chem. Lett., 2017, 8, 5338.

11 C. Zeng, H. Qian, T. Li, G. Li, N. L. Rosi, B. Yoon, R. N. Barnett, R. L. Whetten, U. Landman and R. Jin, Total structure and electronic properties of the gold nanocrystal $\mathrm{Au}_{36} \mathrm{SR}_{24}$, Angew. Chem., Int. Ed., 2012, 51, 13114.

12 S. Tian, et al., Structural isomerism in gold nanoparticles revealed by X-ray crystallography, Nat. Commun., 2015, 6, 8667. 
13 H. Hakkinen, The gold-sulfur interface at the nanoscale, Nat. Chem., 2012, 4, 443.

14 O. Voznyy, J. J. Dubowski, J. T. Yates and P. Maksymovych, The role of gold adatoms and stereochemistry in self-assembled methylthiolate on Au(111), J. Am. Chem. Soc., 2009, 131, 12989.

15 P. Maksymovych, D. C. Sorescu and J. T. Yates, Gold-adatommediated bonding in self-assembled short-chain alkanethiolate species on the Au(111) surface, Phys. Rev. Lett., 2006, 97, 4.

16 Q. Guo and F. Li, Self-assembled alkanethiol monolayers on gold surfaces: resolving the complex structure at the interface by STM, Phys. Chem. Chem. Phys., 2014, 16, 19074.

17 L. Tang, F. Li, W.-C. Zhou and Q. Guo, The structure of the methylthiolate and ethylthiolate monolayers on $\mathrm{Au}(111)$ : absence of the (root $3 \times$ root 3) R30 degrees phase, Surf. Sci., 2012, 606, L31.

18 L. Tang, F. Li and Q. Guo, Complete structural phases fro self-assembled methylthiolate monolayers on $\mathrm{Au}(111)$, J. Phys. Chem. C, 2013, 117, 21234.

19 F. Li, L. Tang, O. Voznyy, J. Gao and Q. Guo, The striped phases of ethylthiolate monolayers on the $\mathrm{Au}(111)$ surfaces: a scanning tunneling microscopy study, J. Chem. Phys., 2013, 138, 194707.

20 J. Gao, F. Li and Q. Guo, Balance of forces in self-assembled monolayers, J. Phys. Chem. C, 2013, 117, 24985.

21 P. Maksymovych, O. Voznyy, D. B. Dougherty, D. C. Sorescu and J. T. Yates Jr, Gold adatoms as a key structural component in self-assembled monolayers of organosulfur molecules on $\mathrm{Au}(111)$, Prog. Surf. Sci., 2010, 85, 206-240.

22 S. Clair, Y. Kim and M. Kawai, Coverage-dependent formation of chiral ethylthiolate-Au complexes on $\mathrm{Au}(111)$, Langmuir, 2010, 27, 627-629.

23 Y. Yourdshahyuan and A. M. Rappe, Structure and energetics of alkanethiol adsorption on the $\mathrm{Au}(111)$ surface, J. Chem. Phys., 2002, 117, 825.

24 S. Rousset, V. Rapain, G. Baudot, Y. Garreau and J. Lecoeur, Self-ordering of $\mathrm{Au}(111)$ vicinal surfaces and application to nanostructure organized growth, J. Phys.: Condens. Matter, 2003, 15, S3363-S3392.

25 L. Tang, X. Zhang and Q. Guo, Site-specific chemistry directed by a bi-functional nanostructured surface, Langmuir, 2010, 26, 4860.

26 J. Gao, F. S. Li and Q. Guo, Mixed methyl- and propylthiolate monolayers on the $\mathrm{Au}(111)$ surface, Langmuir, 2013, 29, 11082.

27 G. E. Poirier, Characterization of organosulfur molecular monolayers on $\mathrm{Au}(111)$ using scanning tunneling microscopy, Chem. Rev., 1997, 97, 1117.

28 R. G. Nuzzo, L. H. Dubois and D. L. Allara, Fundamental studies of microscopic wetting on organic-surfaces. 1. Formation and structural characterization of a self-consistent series of polyfunctional organic monolayers, J. Am. Chem. Soc., 1990, 112, 558. 